\title{
Emerging Fungal Infections in Cancer Patients- A Brief Overview
}

\section{Suganthini Krishnan $\mathbb{N}^{*}$}

Department of Medicine, Division of Infectious Diseases, John D. Dingell VA Medical Center, Wayne State University, Detroit, MI, USA

${ }^{*}$ Corresponding author: Suganthini Krishnan N, Department of Medicine, Division of Infectious Diseases, John D. Dingell VA Medical Center, Wayne State University, Detroit, MI, USA, Tel: 313-576-3057; Fax: 313-576-1242; E-mail: suganthini.krishnannatesan@va.gov

Received date: June 21, 2016; Accepted date: July 26, 2016; Published date: August 06, 2016

Citation: Krishnan NS (2016) Emerging Fungal Infections in Cancer Patients- ABrief Overview Med Mycol Open Access 3: 12. doi: 10.21767/2471-8521.100016.

Copyright: @ 2016 Krishnan NS. This is an open-access article distributed under the terms of the Creative Commons Attribution License, which permits unrestricted use, distribution, and reproduction in any medium, provided the original author and source are credited.

\section{Abstract}

The incidence of invasive fungal infections in immunocompromised cancer patients has continued to increase over the last few decades. With an increase in the use of broad spectrum antifungal prophylaxis, changes in transplant protocols, novel chemotherapeutic agents as conditioning regimens, use of potent $B$ and $T$ cell immunosuppressive agents to decrease the incidence of graft virus host disease, and an increase in the immunocompromised pool of patients, there has been a noticeable shift in the epidemiology of invasive fungal infections. Empiric therapy targeting frequently reported or expected fungal pathogens may fail because of the emergence of rare pathogens and a change in risk factors in the host. Emergence of rare yeasts and molds, often poses a challenge to clinicians involved in the care of these patients. This article provides a brief overview of rare fungal pathogens that have emerged in cancer patients over the last several years. It is meant to deliver an integrated and strategical approach for the diagnosis and management of these unique infections, from a clinical perspective.

Keywords Emerging molds; Non-fumigatus Aspergillus; Zygomycetes; Scedosporium; Fusarium; Yeast-like fungi; NonCandida yeast; Trichosporon; Phaeohyphomycoses; Voriconazole; Posaconazole; Amphotericin B; Isavuconazole

\section{Introduction}

Invasive fungal infections (IFI) continue to pose a therapeutic challenge in immunocompromised cancer patients and are associated with a high incidence of morbidity and mortality. Despite several advances in the field of fungal diagnostics and therapeutics, mortality trends from IFI have continued to rise over the last two decades with a $35.7 \%$ increase from 1980 to 1997 [1]. Historically, Candida and Aspergillus species have accounted for most of yeast and mold infections reported in immunocompromised cancer patients. However, in the last decade, there has been a perceptible emergence of non-Candida yeast, non-fumigatus Aspergillus species, Fusarium spp, Scedosporium spp, Zygomycetes and the Phaeohyphomycoses from various cancer centers around the world [2-9]. The reasons for this observation are purely speculative at this time; some of the conceivable explanations include the introduction of novel, highly potent chemotherapeutic and immunosuppressive agents that have prolonged the survival of cancer patients and the antifungal prophylactic protocols, implemented at various transplant centers [10]. Specific risk-based antifungal strategies have been implemented by various transplant centers leading to the use of voriconazole and echinocandins as prophylactic and pre-emptive therapies $[10,11]$. Fungal diagnostics and therapeutics remains a challenge. This review provides a summary of the emerging fungal pathogens, the epidemiological shift, diagnostic clues and management options that are currently available to treat these infections [12-15].

\section{Yeast-like Fungi}

\section{Trichosporon species}

Trichosporon is an extremely rare but important pathogen in immunocompromised cancer patients. This group includes 8 different species with variable antifungal susceptibilities. They were previously grouped under $T$. beigelii of which $T$. asahii is the most common species associated with disseminated trichosporonosis. Unlike most fungal pathogens, in addition to being distributed widely in the environment, they are normal commensals of the human respiratory and gastrointestinal tract as well. Risk factors overlap with most other opportunistic fungi the most significant ones being hematological malignancy, hematopoietic stem cell transplantation and hemochromatosis or other iron overload conditions. Trichosporon often coexists with other opportunistic fungi, and has been frequently associated with fungemia and skin lesions. It has also been associated with catheter-related infections, pneumonia, prosthetic valve infections, peritoneala-dialysis catheter associated peritonitis, prosthetic joint infections, brain abscess, ventriculo-meningeal infections, and disseminated infections. Studies have reported breakthrough infections in patients treated with echinocandins 
and therefore should be considered in patients who have recently been on echinocandins [16-21].

Diagnosis is met with difficulties as BAL and tissue cultures are frequently negative. Blood cultures occasionally are positive for yeast-like forms and hence diagnosis relies on histopathological examination of tissue specimens (hyphae and pseudohyphae with characteristic arthroconidia), serum galactomannan antigen or 1,3 beta D-glucan testing. Molecular diagnosis using pan fungal PCR assay and the recently introduced MALDI-TOF (Matrix Assisted Laser Desorption Ionization-Time of Flight Mass Spectrometry) appear promising [22-24].

Management strategies for disseminated trichosporonosis have not been clearly defined due to paucity of clinical data. Most species produce biofilm that renders them 1000 times more resistant to triazoles and polyenes. Amphotericin $B$ fluconazole, miconazole and ketoconazole have been used in the past with variable success rates. Breakthrough infections have been reported during antifungal therapy with either voriconazole or echinocandin $[25,26]$.

Voriconazole has been used with limited success. Chen et al. have reported treatment with a combination of liposomal $\mathrm{AMB}$ and caspofungin with a successful outcome in a patient who had breakthrough infection on voriconazole [27]. Data with posaconazole is insufficient except for a liver transplant patient with disseminated $T$. mucoides who responded well to posaconazole therapy [28]. Several antifungal combinations of polyene with azoles (ketoconazole, miconazole and fluconazole) or 5-flucytosine, have been reported in literature with variable success rates [29-33].

\section{Rhodotorula species}

Rhodotorula rubra and $R$. mucilaginosa are red yeasts that were long considered to be non-pathogenic saprophytic organisms. However, in the past decade, reports of invasive infections including catheter related fungemia, skin infections, peritonitis, meningitis, endocarditis, keratitis and endophthalmitis have emerged in cancer patients. Predisposing risk factors in a descending order of frequency include the presence of an indwelling central venous catheter, hematological or solid organ cancers, corticosteroid or cytotoxic chemotherapy [34]. In fact, a 9-year retrospective study by Capoor et al. reported that prolonged hospitalization for $>1$ month and $>1$ month of exposure to broad spectrum antibiotics were major predisposing fatoirs for infection. In a recent review by Tuon et al. the most common pathogen was Rhodotorula mucilaginosa, $79 \%$ were catheter related fiungemias, $87 \%$ of patients had underlying cancer or immunosuppression and most patients responded well to amphotericin therapy with or without catheter removal $[35,36]$. Recovery of neutropenia and a reduction in immunosuppressive therapy play a crucial role in management. Report of successful treatment with catheter removal alone without antifungal therapy has also been reported. In general, Trichosporon is considered to be a lowvirulent pathogen with an associated mortality rate of $\sim 12 \%$ [37]. Diagnosis is by standard fungal culture methods and panfungal PCR. These fungi are capable of producing biofilms that make more resistant to antifungal therapy. Polyenes are the drug of choice with $A M B$ exhibiting the lowest

MIC of $\leq 1 \mathrm{mcg} / \mathrm{ml}$, compared to azoles and echinocandins that have poor activity against these yeasts (Voriconazole MIC90 $4 \mathrm{mcg} / \mathrm{ml}$; Caspofungin MIC $\geq 64 \mathrm{mcg} / \mathrm{ml}$; Fluconazole $>4 \mathrm{mcg} / \mathrm{ml}$ ). Breakthrough infections with Rhodotorula species have occurred in patients receiving azole or echinocandin prophylaxis that is partly attributed to its intrinsic resistance to these antifungal agents. Posaconazole (MIC50 $0.25 \mathrm{mg} / \mathrm{L}$ ) and flucytosine (MIC59 $0.12 \mathrm{mg} / \mathrm{L}$ ) have demonstrated good in vitro activity but data on clinical efficacy is lacking [38-41].

\section{Malassezia furfur}

Classically, these organisms have been associated with pityriasis versicolor and folliculitis. However, in cancer patients, they have been reported to cause invasive infections including catheter-related fungemia and disseminated infections. Most cases have been reported from neonates, children and immunocompromised cancer patients, particularly those requiring parenteral with lipid emulsions. This is likely related to the lipophilic nature of the yeast with lipids presumably providing growth factors required for replication. The pathogenesis involves initial colonization of intravenous catheters with subsequent dissemination [42]. Diagnosis is my standard microbiological culture. Imidazoles and triazoles have a variable in vitro susceptibility pattern with MIC in the range of $0.03-16 \mathrm{mg} / \mathrm{L}$ and cannot be used in serious infections.

Malessezia breakthrough pachydermatitis has been reported on posaconazole prophylaxis in a patient with acute myeloid leukemia [43]. The antifungal drug of choice with the most available clinical data is amphotericin B and catheter removal is in general associated with the best outcome $[44,45]$.

\section{Geotrichum capitatum \\ Blastoschizomyces capitatus)}

(formerly

G. capitatum is a yeast ubiquitous in nature and like Candida spp. is a normal flora of the human respiratory and gastrointestinal tracts and has been isolated from sputum and feces of healthy humans. The mode of acquisition includes inhalation or ingestion. The most important predisposing factors are profound, prolonged neutropenia in hematological malignancies, and broad spectrum antibiotic exposure. Disseminated infections although rare are exclusively reported in immunocompromised patients, particularly those with hematological cancers [46-48]. In a 20-year multicenter study, Giirmenia et al. have reported that $65 \%$ of patients had $A M L$, $76.9 \%$ had concomitant fungemia and the mortality rate was $57.1 \%$ [19]. Diagnosis is by standard microbiological identification and has significantly improved with the introduction of MALDI-TOF and galactomannan assays. Molecular diagnosis based on PCR analysis is under evaluation $[49,50]$. Polyenes and voriconazole have demonstrated good in vitro antifungal activity. Although historically amphotericin $B$ 
has been the drug of choice, clinical success with voriconazole alone or in combination with amphotericin B has been reported in literature. Breakthrough infections in patients on caspofungin prophylaxis have occurred due to its intrinsic resistance to echinocandins. A combination of voriconazole and caspofungin was successfully tried in a case of probable geotrichosis in a leukemic patient. Despite appropriate therapy, geotrichosis has an extremely poor prognosis with a mortality rate approaching $60-75 \%$ [51].

\section{Mold Filamentous Fungi}

\section{Aspergillus species}

A. fumigatus is the most common cause of invasive aspergillosis (IA) followed by $A$. flavus, A. terreus, $A$. niger, $A$. ustus and $A$. lentulus. Several cancer centers around the world have reported the emergence of $A$. niger, $A$. flavus and $A$. terreus over the last several years which is thought to be a consequence of the widespread use of voriconazole prophylaxis in cancer patients. Non- fumigatus Aspergillus spp. have a variable susceptibility pattern to the available antifungal agents [2-4]. A. flavus, $A$. ustus and $A$. lentulus are known to have higher MIC to voriconazole. Also, $A$. terreus is intrinsically resistant to Amphotericin $B$ and this complicates management when voriconazole is used empirically for suspected invasive aspergillosis. There is also a geographic variation in the distribution of various species with $A$. flavus being reported as the predominant pathogen in some institutions in Europe, arid regions of Africa and South East Asia. Clinical syndromes associated with aspergillosis in patients with pre-existing lung disease include allergic pulmonary aspergillosis, chronic necrotizing aspergillosis and aspergilloma. On the contrary, the most common form reported in immunocompromised cancer patients is invasive pulmonary aspergillosis, although it could invade other organs including the central nervous system or cause disseminated infections [52-54]. Earlier studies have reported most cases of IA during the prolonged neutropenic phase in the early postHSCT period (13\% of mismatched allograft recipients) [55]. However, recent observations have noted a shift in the time of occurrence of IA with late-onset disease being increasingly reported in the following groups: (a) unrelated hematopoietic stem cell recipients (HSCT) with graft versus host disease (GVHD) requiring intense immune-suppression with corticosteroid (b) T- cell depleted patients and (c) CMV disease. Among solid organ transplant recipients, invasive pulmonary aspergillosis is most often reported in lung transplant recipients, with single lung transplant recipients at higher risk, where they acquire infection from the nontransplanted colonized lung $[55,56]$.

Diagnosis is frequently made by demonstration of the acute angled septate hyphae from histopathological examination of tissue from tissue biopsy specimens. Bronchoscopy followed by culture of the bronchoalveolar lavage has a poor sensitivity of $30-50 \%$. However because of problems with thrombocytopenia and bleeding risks, invasive techniques are not favored. As a result, non-invasive techniques looking for fungal cell wall components have gained momentum in the recent years. The galactomannan antigen and beta-D glucan tests are being widely used although false positive and negatives remain a problem. PCR analysis for identification of fungal DNA directly from blood would be a valuable diagnostic tool, but requires standardization and validation prior to being implemented in the clinical practice [57-60]. In fact some of the PCR tests have been validated and standardized. Johnson et al. have described the 2 novel tests namely the Aspergillus specific lateral flow device and real time PCR assay that have good sensitivity and specificity [61]. In patients with invasive pulmonary aspergillosis, a combination of high resolution computerized-tomographic scan (CT scan) of the thorax with galactomannan antigen testing of bronchoalveolar fluid (more sensitive than serum) and serum may aid in earlier diagnosis and better outcome. The CT scan findings include pulmonary nodules or infiltrates with halo sign in neutropenic phase and the air crescent sign in the neutrophil recovery phase although none of these are specific for aspergillosis and may be seen with other molds as well [54-58].

The drugs currently approved for the treatment of invasive aspergillosis include polyenes voriconazole and the latest addition isavuconazole. A randomized, double-blind comparison clinical trial for treatment of invasive aspergillosis found that the efficacy of isavuconazole was non-inferior to that of voriconazole. Drug-related toxicities have made $A M B$ a less favorable agent and it has been replaced by VCZ which is currently indicated as first line therapy for IA. The landmark study that led to approval of VCZ for primary therapy involved 277 patients with 144 in the VCZ group and 133 in the AMB deoxycholate group. At the end of 12 weeks, the response rate was $52.8 \%$ of patients in the VCZ group and $31.6 \%$ of patients in the $A M B$ group.

Since the publication of this study, VCZ has been widely accepted as the drug of choice for the treatment of IA [62]. In the ambiload trial, voriconazole was compared with high-dose liposomal amphotericin B $(10 \mathrm{mg} / \mathrm{kg} /$ day $)$ and the authors concluded that there was no beneficial effect with administration of a high loading dose of liposomal AMB and in fact, the high dose was associated with an increased risk of nephrotoxicity. With its better toxicity profile voriconazole is preferred to $A M B$ or its lipid formulations. Nonetheless, Amphotericin $B$ (conventional or its lipid formulations) may be preferred in certain clinical situations as follows: (1) previous use of a mold active agent for prophylaxis or empiric therapy; (2) concomitant use of drugs with major interactions with VCZ (cyp450 interaction) such as tacrolimus, sirolimus, rifampin or warfarin; (3) hepatic impairment (4) adverse effects to voriconazole (5) high suspicion for zygomycoses or other mold that may not be susceptible to VCZ (6) presence of cardiac risk factors such as prolonged QT interval or cardiomyopathy [63]. Posaconazole although approved for prophylaxis, has been widely used as salvage therapy for refractory invasive aspergillosis $[64,65]$. Isavuconazole is the novel addition to the antifungal armamentarium with several advantages over voriconazole such as (1) fewer drug interactions (2) fewer adverse effects (3) availability of water soluble parenteral 
formulation (4) excellent oral bio-availability and (5) excellent pharmacokinetic parameters [66,67].

Echinocandins, although approved exclusively for salvage therapy, have also been evaluated for primary therapy against IA. The overall response rate to caspofungin or micafungin monotherapy for invasive aspergillosis ranges from 50-56\% and has been dismal. Although echinocandins appear intuitively promising, given their unique mechanism of action on the cell wall glucan, clinical data so far, has recommended against the use of echinocandin monotherapy. A study from Denning et al., that evaluated the efficacy of micafungin either alone or in combination showed a response rate of $50 \%$. However, the group of patients that received micafungin alone was small $(n=23)$ [15]. A recent extensive review by MartinPena et al. discussed the use of various antifungal combinations, including echinocandin combination with either amphotericin B or voriconazole based on literature review [68].

With several in vitro and in vivo animal studies demonstrating synergy with the combination of voriconazole and echinocandins, studies on combination therapy for IA have gained momentum over the last few years. Combinations of voriconazole plus caspofungin, liposomal $A M B$ plus caspofungin (Combistrat trial) and micafungin plus $A M B$ or triazoles have been tried with reasonably good response rates [13]. Despite lack of supporting data, sequential addition of echinocandin to voriconazole is common practice in patients with refractory/progressive IA with the presumption that if offers better efficacy. A randomized double blind multicenter clinical trial assessed the safety and efficacy of voriconazole and anidulafungin compared with voriconazole monotherapy for treatment of IA. Authors concluded that combination therapy with voriconazole plus anidulafungin led to higher survival in subgroups of patients with IA, compared to voriconazole monotherapy [69].

\section{Scedosporium species}

S. apiospermum (the asexual form of $P$. boydii) and $S$. prolificans are ubiquitous in the environment, found in soil and decaying organic matter and are the most common species associated with infections in cancer patients. The spectrum of infection in immunocompromised hosts includes pneumonia, invasive fungal sinusitis, cutaneous infections, osteomyelitis, brain abscess, endophthalmitis and disseminated scedosporiosis with septic shock. It has been associated with cutaneous or bone infections in immunocompetent hosts and in near-drowning incidents in normal hosts as well. Scedosporium infections account for about $20 \%$ of all nonaspergillus mold infections in solid-organ transplant patients with an associated mortality rate of 50\% [70-78].

In a recent study by Husain et al. involved 270 patients, 57 cases were seen in organ transplant recipients, 23 cases in HSCT recipients and 190 cases in non-transplant recipients. The most common risk factors reported include neutropenia and immunesuppression in the post-transplant period. Although S. apiopsermum was the most common pathogen overall, S. prolificans was predomionantly seen in HSCT, had a higher incidence of fungemia $(57 \%$ versus $8 \%$ in $S$. apiospermum), high risk for dissemination and was associated with a higher mortality [79]. A retrospective study conducted over a 12 year period in Australian patients reported an incidence of $33.3 \%$ of Scedosporium infections and the commonest comorbidities included hematological malignancies (46.3\%), diabetes mellitus (23.5\%) and chronic pulmonary diseases (16\%) [80]. Clinical presentation, imaging findings and histopathology have a marked resemblance to that of invasive aspergilllosis. In the absence of a positive culture, this has clinical implications as $S$. apiospermum is universally resistant to amphotericin $B$ and if used empirically for presumed IA, it would be ineffective. Also, S. prolificans exhibits a very poor susceptibility profile being resistant to most currently available antifungal agents. It is also noteworthy that unlike Aspergillus spp., Scedosporium spp. has adventitial forms capable of in vivo sporulation that promotes rapid hematogenous dissemination.

Microbiological diagnosis of Scedosporium infections has relied on conventional culture and morphological characterization of isolates from histopathological examination of tissue. The latest introduction of MALDI-TOF mass spectrometry has aided in the rapid diagnosis of these infections [81]. Antigen based diagnostic tests and molecular tools for microbiological detection are under investigation.

All species of Scedopsorium are resistant to polyenes. The currently available triazoles have good efficacy against $S$. apiospermum. Cross resistance has been reported among all triazoles except posaconazole. Echinocandins have demonstrated good in vitro antifungal activity against $S$. apiospermum, although clinical data is insufficient. On the contrary, S. prolificans is resistant to most currently available antifungal agents including the second generation triazoles and poses an enormous challenge to the managing physician. Several novel triazole agents are currently under investigation and are in the pipeline. The combination of voriconazole and terbinafine was efficacious with successful outcome in an orthopedic infection [82]. Most published reports are on the successful use of voriconazole, data on posaconazole use is scarce, but it has been used with favorable outcome [83-86]. A reduction in immune-suppressive therapy and surgical resection of localized lesions if feasible must be an integral part of management of these difficult to treat fatal infections [84]. Trubiano et al. have used a combination of voriconazole with adjunctive miltefosine and a cyp2C19 inhibitor namely cimetidine, with success, in a patient with disseminated $S$. prolificans infection who was a rapid metabolizer [87].

\section{Fusarium species}

Among the 50+ species reported so far only F. solani (most common), F. oxysporum, F. moniliforme, F. verticillioides, $F$. dimerum and $F$. proliferatum are known to be pathogenic in humans. The principal portal of entry is the respiratory tract with a wide spectrum of presentation that includes pneumonia, cutaneous infections, brain abscess, invasive fungal sinusitis, fungemia or disseminated infections, depending on the immune status of the host. Based on data 
from in vivo animal studies $F$. solani is considered to be the most virulent of all fungal pathogens reported. Published data suggests that high-dose corticosteroid therapy, severe T-cell dysfunction and prolonged neutropenia are significant risk factors associated with disseminated fusariosis. The risk factors, patient characteristics, clinical features and histopathology, overlap with those of Aspergillus and Scedosporium spp. Differentiating the two pathogens in the clinical setting may be challenging for the physician in the already perplexing field of fungal diagnostics, particularly in the absence of culture results. Although Fusarium infections are relatively less common than IA, the presence of fungemia, cellulitis, onychomycosis or cutaneous catheter-related infections should be considered as subtle clues to the possible diagnosis of fusariosis [88-95]

A 13-year (2002-14) review by Stempel et al. reported that the most common underlying conditions were hematological malignancies (87\%), juvenile idiopathic arthritis (7\%), and 3rd degree burns (7\%). Of these $53 \%$ were skin and soft tissue infections, $27 \%$ were associated with febrile neutropenia, $13 \%$ were pneumonia and $7 \%$ of reported cases were septic arthritis. A vast majority of infections have been reported in HSCT recipients ( 6 per 1000 HSCTs; 20 per 1000 in mismatched HSCTs) compared to solid organ transplant recipients [96]. In a study of 84 patients with hematological diseases by Nucci et al. $56 \%$ of cases occurred in patients with acute leukemia and most (86\%) of them were neutropenic at the time of diagnosis. There was a trimodal distribution demonstrated in HSCT patients with the first peak in the early post-transplant period, the second peak in the 3rd month seen in patient receiving corticosteroid therapy for acute GVHD and the 3 rd peak $>1$ year post transplant in patients with chronic GVHD on corticosteroids [97].

Early diagnosis cannot be overemphasized as it greatly impacts mortality. Importantly, blood cultures are positive in $82 \%$ of disseminated infections. Growth of Fusarium spp. can be detected in blood cultures 10 to $35 \mathrm{hrs}$ earlier, when fungal medium was used particularly in the presence of low fungal inoculums [98]. Differentiating it from Aspergillus spp. and other hyalohyphomycoses is a persistent issue as tissue cultures are frequently negative. Diagnosis is based on histopathological examination of tissue specimens where the presence of acute dichotomously branching septate hyphae along with yeast-like structures in tissue (adventitious sporulation) is strongly suggestive of fusariosis. A positive 1, 3, beta $\mathrm{D}$-glucan test with a negative galactomannan antigen test in the appropriate clinical context is highly suggestive of fusariosis. MALDI-TOF mass spectrometry has good sensitivity and specificity $[98,99]$. Molecular testing using PCR is still in development and not standardized.

Fusarium species exhibit differential susceptibility patterns to various antifungal agents available. Clinical experience is limited and most data available are from retrospective analysis and salvage studies. F. solani and F. verticilloides are usually resistant to triazoles whereas $F$. oxysporum and $F$. moniliforme may be susceptible to voriconazole and posaconazole. Most species are resistant to polyenes [100-103]. Nucci et al. performed a retrospective analysis of 84 patients with hematologicl malignancies and invasive fusariosis who received amphotericin $B$ deoxycholate or a lipid formulation of amphotericin $\mathrm{B}$. The response rate to the lipid formulation was $46 \%$ in comparison to amphotericin B deoxycholate (32\%) but it was not statistically significant. However, the 90-day mortality rate from the time of diagnosis was 80\% [97]. Another retrospective review of HSCT recipients demonstrated a poor outcome to treatment with either regular $A M B$, lipid formulation of $A M B$ or caspofungin, reaffirming the previous study. On the other hand, there were few other salvage studies one using amphotericin B ( $n=26$ with response rate of $46 \%$ ) and the other using voriconazole $(n=11$ with a response rate of $45 \%)$. The better outcome seen in these salvage studies is likely related to a selection bias, with patients surviving long enough to receive salvage therapy having a better prognosis to begin with in comparison to the other group. Posaconazole (minimal data) has demonstrated modest success rates when used as salvage therapy in 21 patients of whom $76 \%$ had hematological malignancy and $38 \%$ were neutropenic at the time of diagnosis. The overall success rate was $48 \%$ with better results seen in localized disease as compared to disseminated infection $[100,101]$. Combination of AMB with caspofungin, VCZ or terbinafine and VCZ plus terbinafine has been tried with variable success rates $[102,103]$.

Granulocyte transfusions, G-CSF and GM-CSF have also been employed as adjuncts in combination with antifungal agents. In practice, several different combination therapies are being advocated based on case reports and anecdotal data. The paucity of clinical data emphasizes the need for more in vivo studies and pending further studies, a definite recommendation on combination therapy cannot be made at this time.

\section{Zygomycetes}

Zygomycosis is an aggressive and often lethal infection that occurs most commonly in immunocompromised patients representing $5-20 \%$ of all invasive fungal infections in this population. Illness is rapidly progressive with angioinvasion and tissue infarction (hallmark features of this disease). Mortality despite appropriate therapy remains high. The clinical syndrome in human disease encompasses rhinocerebal, pulmonary, cutaneous, gastrointestinal, and disseminated zygomycosis. The most common risk factors in the transplant setting include an underlying myelodysplastic syndrome associated with repeated blood transfusions resulting in an iron overload condition, prolonged neutropenia, diabetes mellitus, chronic GVHD on corticosteroid therapy and recipients of antithymocyte globulin. Etiological agents reported in order of frequency are Rhizopus and Mucor followed by Absidia and Rhizomucor. There has been an emergence of zygomycoses noted in various transplant centers in the United States and around the world [104-106]. This increased incidence is in part attributable to the prophylactic protocols (triazoles or echinocandins) that offer protection against Candida spp., Aspergillus spp. and Scedosporium spp. This subsequently paves way for colonization of these patients with Zygomycetes leading to 
invasive infection when the host immunity further wanes $[106,107]$.

Most cases of disseminated zygomycoses are diagnosed post-mortem. It cannot be overemphasized that the index of suspicion should be high in selected high-risk patients. Early diagnosis is critical as it directly impacts mortality. The major hurdle in the management of Zygomycoses is the angioinvasive nature of the pathogen with intravascular thrombosis resulting in necrosis that hinders the penetration of antifungal drugs at the site of infection. Urgent surgical debridement is an integral part of management to thwart further dissemination. Hence localized or focal lesions (pulmonary, cutaneous or rhinocerebral) must be aggressively pursued by a diagnostic tissue biopsy, followed (if positive) by surgery. Blood and tissue cultures are virtually always negative. Molecular diagnosis using PCR/RT-PCR is available in certain laboratories. Diagnosis therefore relies on conventional histopathological examination of tissue that demonstrates broad aseptate hyaline hyphae on gomori methanamine silver staining. Examination of frozen sections of tissue at the time of surgical intervention is crucial in order to expedite the diagnosis and initiate appropriate antifungal therapy $[108,109]$.

The optimal treatment for zygomycosis has not been well defined owing to the lack of controlled prospective trials involving this relatively uncommon opportunistic mycosis. Historically polyenes have been the only agents available for treatment of infections due to zygomycetes. Recently, the novel extended spectrum triazole, PCZ has demonstrated good efficacy in vivo, against zygomycetes and is currently being used as salvage therapy for zygomycosis. Based on published literature, both agents seem to have good antifungal efficacy and equivalent survival rates in vivo. A brief review of prospective/retrospective studies, clinical trials and case reports has demonstrated reasonably good success rates in patients when posaconazole was used as salvage therapy [110-112]. Nonetheless, despite the seemingly favorable response rates observed with posaconazole, results from these studies must be interpreted with caution. Importantly, all study patients had received a course of $A M B$ prior to the initiation of PCZ. Therefore there remains a possibility that the favorable outcome observed in these cases may have been a consequence of either sequential therapy ( $\mathrm{AMB}$ followed by $P C Z)$ or a synergistic effect owing to the long half-life of $A M B$ and not exclusively due to PCZ by itself [110].

An open-label trial that studied primary as well as salvage therapy of invasive mucormycosis showed that efficacy of isavuconazole was similar to amphotericin $B$ and posaconazole, with a better safety profile compared to other antifungal agents. The study included a total of 37 patients with mucormycosis who received isavuconazole for a median of 84 days. All-cause crude mortality recorded on day 42 of treatment was $33 \%$ and similar to the group that was treated with amphotericin B [111].

The role of echinocandins against zygomycetes has not been well established. Despite poor in vitro activity, clinical success with a combination of polyene and micafungin has been reported. This is thought to be related to the possible anti- inflammatory property of echinocandins. More studies are needed before any further recommendations can be made regarding the routine use of echinocandins as adjunctive therapy for zygomycoses [112].

Novel iron chelators that are hydroxypyridinone derivatives such as deferiprone and deferasirox, unlike deferoxamine, do not exhibit a higher affinity for iron and therefore deprive fungi of iron. These agents have been widely tested in vitro and in vivo and are being used as adjuncts on a compassionate basis for treating life threatening zygomycoses [113]. Clinical trials evaluating the antifungal efficacy of this interesting combination are ongoing. Other adjunctive therapies that have received significant consideration in the past have involved the use of hyperbaric oxygen therapy, cytokines and colony stimulating growth factors. However their role has not yet been adequately studied and available data is insufficient to warrant their use in the management of these infections.

\section{Phaeohyphomycetes}

Dematiaceous or black molds are a heterogenous group of organisms that obtained their nomenclature from the presence of a brown-black pigment (melanin) incorporated in their cell wall. The genera include Bipolaris, Alternaria, Curvularia, Exophiala, Exserohilum and Wangiella species. Bipolaris spicifera is the etiological agent frequently reported from immunocompromised cancer patients. Although considered to be less virulent in general, it is also known to cause serious disseminated infections in cancer patients. Being distributed widely in soil, wood and decomposing plant debris, infections are airborne, involve the skin or the sinuses and are commonly reported from tropical countries and usually affect immunocompetent individuals. A recent review suggested that the number of case reports has increased 10 -fold over the last 30 years. Disseminated infection is uncommon, but its incidence is increasing, particularly among immunocompromised individuals. Some are considered to be neurotropic, including Cladophialophora bantiana, Ramichloridium mackenziei, and Wangiella dermatitidis [114-119].

A multi-state outbreak of spinal infections was reported in 2012, secondary to contamination of methylprednisolone acetate with the black mold, Exserohilum rostratum. More than 13,000 patients were exposed to the potentially contaminated drug, 741 were confirmed to be drug-related infections, and 55 deaths were recorded. Fatal meningitis and localized epidural, paraspinal, and peripheral joint infections occurred. Diagnosis is made on the observation of yeast-like cells, hyphae and pseudohyphae alone or in combination and the dark-wall seen on special staining procedures. Blood and tissue cultures have poor sensitivity and there are no antigen based or standardized PCR tests currently available. The above mentioned outbreak cases were diagnosed by immunehistochemstry and PCR from infected tissue [120]. With the rare occurrence of invasive infections in cancer patients, available data are scarce to come up with any reliable conclusions pertaining to therapy. However, published 
literature suggests that patients benefit from a combination of both surgical and medical intervention.

The lack of standardized antifungal susceptibility testing with the pattern of heterogenous antifungal susceptibility pattern further complicates the management of infections due to black molds. Antifungal agents known be efficacious include AMB-deoxycholate and itraconazole. Data on use of voriconazole and posaconazole is scarce. Itraconazole has been the most frequently used agent; the role of lipid formulations of $A M B$ is unclear; and voriconazole activity approximates that of itraconazole with MICs for both agents in the range of 0.25 to $2 \mathrm{mcg} / \mathrm{ml}$ depending on the genera tested. Posaconazole has demonstrated good in vitro fungicidal activity against Exophiala jeanselmi and in vivo activity against central nervous system infection in an animal model. Fluconazole and echinocandins have no activity against any of the species. The azole in development UR 9825 has good in vitro activity against $E$. spinifera. Surgical resection of localized lesions with a reduction in immunosuppression is recommended in all cancer patients if feasible [121]

\section{Cryptococcus}

C. neoformans and C. gattii are the two major species associated with cryptococcal infections. C. gattii was the etiologocal agent in an outbreak of cryptococcosis in immunocompetent humans and animals on Vancouver Island and surrounding areas within Canada and the northwestern United States. Cryptocoocus meningitis and meningoencephalitis are frequently seen in human immunodeficiency virus infections and relatively less common in immunocompromised cancer patients and hematopoietic stem cell recipients. Our overview here will focus on immunocompromised solid organ transplant recipients, where it is usually reported $\sim 1$ to 2 years post-transplant depending on the intensity of immunosuppression with an incidence rate of $2.8 \%$. Cryotococcal fungemeia has been reported in $25 \%$ of these patiens. Infections frequently involve the lung in $25 \%$ $54 \%$ and in $6 \%-33 \%$ of these patients, the disease is limited to the lungs only. Meningoencephalitis, cryptococcoma and disseminated cryptococcosis have been documented in $52 \%-$ $61 \%$ of patients [122-125].

Diagnosis is made by a positive serum cryptococcal antigen titer and has been documented in 33\%-90\% of transplant recipients with pulmonary cryptococcosis. A negative serum cryptococccal antigen test does not exclude pulmonary cryptococcosis. Patients with central nervous system (CNS) disease or disseminated cryptococcosis have been shown to have a positive serum cryptococcal antigen test $(95 \%$ and $97 \%$, respectively). Therefore, an organ transplant recipient with a positive serum cryptococcal antigen test needs to be worked up for cryptococcal meningitis or dissemination infection [126-129].

For CNS disease, liposomal AmB (3-4 mg/kg/day IV) or ABLC ( $5 \mathrm{mg} / \mathrm{kg} /$ day IV) plus flucytosine $(100 \mathrm{mg} / \mathrm{kg} /$ day in 4 divided doses) for at least 2 weeks. If induction therapy does not include flucytosine, lipid formulation of $A m B$ is indicated for at least 4-6 weeks as induction therapy. A dosage of $6 \mathrm{mg} / \mathrm{kg}$ per day of liposomal AmB must be considered in high-fungal burden disease or relapse. Fluconazole at $400 \mathrm{mg}[6 \mathrm{mg} / \mathrm{kg}$ ] per day for 6-12 months is indicated for mild-to-moderate non-CNS disease. For moderately severe-to-severe non-CNS or disseminated disease, without CNS involvement, treatment is the same as CNS disease. In the absence of any clinical evidence of extrapulmonary or disseminated cryptococcosis, severe pulmonary disease is treated the same as CNS disease. Fluconazole maintenance therapy should be continued for at least 6-12 months $[130,131]$. Management of cryptococccal infection involves decreasing the dosage of steroids and a reduction in immunosuppressive therapy if feasible. Amphotericin deoxycholate is not recommended as first-line therapy in this patient population due to significant nephrotoxicity. Cosmplications including elevated intracranial pressure must be managed by serial lumbar punctures or drainage as appropriate [132]. Also, drug interactions with calcineurin inhibitors could complicate management issues and should be watched for. C.gattii has been reported frequently in immunocompetent hosts, and is rare in AIDS and in transplant recipients. In HIV-negative patients, CNS infection with $C$. gattii has been associated with more complications, delayed response to therapy, and a higher requirement for neurosurgical intervention, compared to $C$. neoformans [133-135]. Given the infrequent occurrence of cryptococcal infections, and questions regarding subclinical infection and recurrence, primary prophylaxis is not indicated at this time, in this patient population.

\section{Conclusions}

The availability of novel chemotherapeutic agents and transplant procedures has significantly improved patient survival rates from cancer. However invasive fungal infections remain a significant therapeutic challenge for physicians and continue to pose a potential threat for patients who survive cancer. Several of the infections once considered rare are being increasingly reported from various cancer centers. With an obvious epidemiological shift, prompt detection of patients at high risk, early diagnosis and appropriate therapy are crucial in the management of these emerging fungal infections. Management of these rare fungi is further complicated due to their heterogenous antifungal susceptibility pattern and intrinsic resistance to the routinely used standard antifungal agents.

The high mortality associated with these rare invasive fungal infections, echoes a long unmet need for more reliable, noninvasive, early diagnostic tools and novel antifungal agents with more potency. Ongoing research in the field of fungal diagnostics and therapeutics would facilitate the introduction of novel, more potent antifungal agents including immunomodulatory therapies that will eventually improve the morbidity and mortality of these serious infections. 


\section{References}

1. Mcneil MM (2001) Trends in mortality due to invasive aspergillosis in the United States 1980-1997. Clinical Infectious Diseases 33: 641-647.

2. Douglas AP, Chen SC, Slavin MA (2016) Emerging infections caused by non-Aspergillus filamentous fungi. Clin Microbiol Infect.

3. Peghin M, Monforte V, Martin-Gomez MT (2016) Epidemiology of invasive respiratory disease caused by emerging nonAspergillus molds in lung transplant recipients. Transpl Infect Dis 18: $70-78$

4. Slavin M, van Hal S, Sorrell TC (2015) Australia and New Zealand Mycoses Interest Group. Invasive infections due to filamentous fungi other than Aspergillus: epidemiology and determinants of mortality. Clin Microbiol Infect 21: 490-510.

5. Chitasombat MN, Kofteridis DP, Jiang Y, Tarrand J, Lewis RE, et al. (2012) Rare opportunistic (non-Candida, non-Cryptococcus) yeast bloodstream infections in patients with cancer. J Infect 64: 68-75.

6. Nucci M (2003) Emerging molds: Fusarium, Scedosporium and Zygomycetes in transplant recipients. Curr Opin Infect Dis 16: 607-612.

7. Michael A, Pfaller, Peter G, Pappas, John R, et al. (2006) Invasive Fungal Pathogens: Current Epidemiological Trends. Clin Infect Dis 43: S3-S14.

8. Malani AN, Kauffman CA (2007) Changing epidemiology of rare mold infections: implications for therapy. Drugs 13: 1803-1812.

9. Silveira F, Nucci M (2001) Emergence of black molds in fungal disease: epidemiology and therapy. Curr Opin Infect Dis 14 679-684.

10. Tong SY, Peleg AY, Yoong J, Handke R, Szer J, et al. (2007) Breakthrough Scedosporium prolificans infection while receiving voriconazole prophylaxis in an allogeneic stem cell transplant recipient Transpl Infect Dis 9: 241-243.

11. Kami M, Machida U, Okuzumi K (2002) Effect of fluconazole prophylaxis on fungal blood cultures: an autopsy-based study involving 720 patients with haematological malignancy. $\mathrm{Br} \mathrm{J}$ Haematol 117: 40-46.

12. Walsh TJ, Kontoyiannis DP (2008) Editorial commentary: what is the role of combination therapy in management of zygomycosis? Clin Infect Dis 47: 372-374.

13. Caillot D, Thiébaut A, Herbrecht R (2007) Liposomal amphotericin $B$ in combination with caspofungin for invasive aspergillosis in patients with hematologic malignancies: a randomized pilot study (Combistrat trial). Cancer 110: 2740-2746.

14. Chamilos G, Kontoyiannis DP (2006) The rationale of combination antifungal therapy in severely immunocompromised patients: empiricism versus evidencebased medicine. Curr Opin Infect Dis 4: 380-385.

15. Kontoyiannis DP, Ratanatharathorn V, Young JA (2009) Micafungin alone or in combination with other systemic antifungal therapies in hematopoietic stem cell transplant recipients with invasive aspergillosis. Transpl Infect Dis 11: 89-93.
16. Suzuki K, Nakase K, Kyo T (2010) Fatal Trichosporon fungemia in patients with hematologic malignancies. Eur J Haematol 84: 441-447.

17. Girmenia C, Pagano L, Martino B (2005) Invasive infections caused by Trichosporon species and Geotrichum capitatum in patients with hematological malignancies: a retrospective multicenter study from Italy and review of the literature. J Clin Microbiol 43: 1818-1828.

18. Kumar A, Udayakumaran S, Babu R (2015) Trichosporon asahii infection presenting as chronic meningo-ventriculitis and intra ventricular fungal ball: a case report and literature review. Mycoses 58: 99-103.

19. Girmenia C, Pagano L, Martino B (2005) GIMEMA Infection Program. Invasive infections caused by Trichosporon species and Geotrichum capitatum in patients with hematological malignancies: a retrospective multicenter study from Italy and review of the literature. J Clin Microbiol 43: 1818-1828.

20. Liao Y, Lu X, Yang S, Luo Y, Chen Q, et al. (2015) Epidemiology and Outcome of Trichosporon Fungemia: A Review of 185 Reported Cases From 1975 to 2014. Open Forum Infect Dis 2: 141.

21. Heslop OD, Nyi Nyi MP, Abbott SP, Rainford LE, Castle DM, et al. (2011) Disseminated trichosporonosis in a burn patient: meningitis and cerebral abscess due to Trichosporon asahii. J Clin Microbiol 49: 4405-4408.

22. Arendrup MC, Boekhout T, Akova M, Meis JF, Cornely OA, et al. (2014) European Society of Clinical Microbiology and Infectious Diseases Fungal Infection Study Group; European Confederation of Medical Mycology. ESCMID and ECMM joint clinical guidelines for the diagnosis and management of rare invasive yeast infections. Clin Microbiol Infect Suppl 3:76-98.

23. Almeida Júnior JN, Song AT, Campos SV (2014) Invasive Trichosporon infection in solid organ transplant patients: a report of two cases identified using IGS1 ribosomal DNA sequencing and a review of the literature. Transpl Infect Dis 16: 135-140.

24. Kushima H, Ishii H, Komiya K, Tokimatsu I, Kadota J (2013) Prognostic significance of serum $\beta$-d-glucan levels in 78 patients with Trichosporon fungemia. Int J Infect Dis 17: e134-135.

25. Yang MF, Gao HN, Li LJ (2014) A fatal case of Trichosporon asahii fungemia and pneumonia in a kidney transplant recipient during caspofungin treatment. Ther Clin Risk Manag 10: 759-762.

26. Matsue K, Uryu H, Koseki M, Asada N, Takeuchi M (2006) Breakthrough trichosporonosis in patients with hematologic malignancies receiving micafungin. Clin Infect Dis 42: 753-757.

27. Chen J, Chen F, Wang Y, Yang LY, Miao M, et al. (2014) Use of combination therapy to successfully treat breakthrough Trichosporon asahii infection in an acute leukemia patient receiving voriconazole. Med Mycol Case Rep 6:55-57.

28. Lacasse A, Cleveland KO (2009) Trichosporon mucoides fungemia in a liver transplant recipient: case report and review. Transpl Infect Dis 2009 11: 155-159.

29. Cornely OA, Cuenca-Estrella M, Meis JF, Ullmann AJ (20140 European Society of Clinical Microbiology and Infectious Diseases (ESCMID) Fungal Infection Study Group (EFISG) and European Confederation of Medical Mycology (ECMM) 2013 joint guidelines on diagnosis and management of rare and emerging fungal diseases. Clin Microbiol Infect Suppl 3:1- 4 
30. Fournier S, Pavageau W, Feuillhade M (2002) Use of voriconazole to successfully treat disseminated Trichosporon asahii infection in a patient with acute myeloid leukaemia. Eur J Clin Microbiol Infect Dis 21: 892-896.

31. Bassetti M, Bisio F, Di Biagio A (2004) Trichosporon asahii infection treated with caspofungin combined with liposomal amphotericin B. J Antimicrob Chemother 54: 575-577.

32. Hosokawa K, Yamazaki H, Mochizuki K (2012) Successful treatment of Trichosporon fungemia in a patient with refractory acute myeloid leukemia using voriconazole combined with liposomal amphotericin B. Transpl Infect Dis 14: 184-187.

33. Asada $N$, Uryu H, Koseki M, Takeuchi M, Komatsu $M$, et al. (2006) Successful treatment of breakthrough Trichosporon asahii fungemia with voriconazole in a patient with acute myeloid leukemia. Clin Infect Dis 43: 39-41.

34. Miglietta F, Letizia Faneschi M, Braione A (2015) Central Venous Catheter-related Fungemia caused by Rhodotorula glutinis. Med Mycol J 56: E17-9.

35. Capoor MR, Aggarwal S, Raghvan C, Gupta DK, Jain AK, et al. (2014) Clinical and microbiological characteristics of Rhodotorula mucilaginosa infections in a tertiary-care facility. Indian J Med Microbiol 32: 304-309.

36. Spiliopoulou A, Anastassiou ED, Christofidou M (2012) Rhodotorula fungemia of an intensive care unit patient and review of published cases. Mycopathologia 174: 301-309.

37. Forés R, Ramos A, Orden B (2012) Rhodotorula species fungaemia causes low mortality in haematopoietic stem-cell transplantation. A case report and review. Mycoses 55: 158-162.

38. Menon S, Gupta HR, Sequeira R (2014) Rhodotorula glutinis meningitis: a case report and review of literature. Mycoses 57: 447-451.

39. Lunardi LW, Aquino VR, Zimerman RA, Goldani LZ (2006) Epidemiology and outcome of Rhodotorula fungemia in a tertiary care hospital. Clin Infect Dis 43: 60-63.

40. Tuon FF, Costa SF (2008) Rhodotorula infection A systematic review of 128 cases from literature. Rev Iberoam Micol 25: 135-140.

41. Tuon FF, de Almeida GM, Costa SF (2007) Central venous catheter-associated fungemia due to Rhodotorula spp-a systematic review. Med Mycol 45: 441-447.

42. Barber GR, Brown AE, Kiehn TE, Edwards FF, Armstrong D (1993) Catheter-related Malassezia furfur fungemia in immunocompromised patients. Am J Med 95: 365-370.

43. Cannizzo FT, Eraso E, Ezkurra PA (2007) Biofilm development by clinical isolates of Malassezia pachydermatis. Med Mycol 45: 357-361.

44. Curvale-Fauchet N, Botterel F, Legrand P, Guillot J, Bretagne S (2004) Frequency of intravascular catheter colonization by Malassezia spp. in adult patients. Mycoses 47: 491-494.

45. Morrison VA, Weisdorf DJ (2000) The spectrum of Malassezia infections in the bone marrow transplant population. Bone Marrow Transplant 26: 645-648.

46. Del Principe MI, Sarmati L, Cefalo M (2016) A cluster of Geotrichum clavatum (Saprochaete clavata) infection in haematological patients: a first Italian report and review of literature. Mycoses.
47. Trabelsi H, Neji S, Gargouri L (2015) Geotrichum capitatum septicemia: case report and review of the literature. Mycopathologia 179: 465-469.

48. Camus V, Thibault ML, David M (2014) Invasive Geotrichum clavatum fungal infection in an acute myeloid leukaemia patient: a case report and review. Mycopathologia 177: 319-324.

49. Miglietta F, Vella A, Faneschi ML (2015) Geotrichum capitatum septicaemia in a haematological patient after acute myeloid leukaemia relapse: identification using MALDI-TOF mass spectrometry and review of the literature. Infec Med 23: 161-167.

50. Bonini A, Capatti C, Parmeggiani M, Gugliotta L, Micozzi A, et al. (2008) Galactomannan detection in Geotrichum capitatum invasive infections: report of 2 new cases and review of diagnostic options. Diagn Microbiol Infect Dis 62: 450-452.

51. Fianchi L, Montini L, Caira M, Voso MT, Maviglia R, et al. (2008) Combined voriconazole plus caspofungin therapy for the treatment of probable Geotrichum pneumonia in a leukemia patient. Infection 36: 65-67.

52. Cornely OA (2008) Aspergillus to Zygomycetes: causes, risk factors, prevention, and treatment of invasive fungal infections. Infection 36: 296-313.

53. Nivoix Y, Velten M, Letscher-Bru V (2008) Factors associated with overall and attributable mortality in invasive aspergillosis. Clin Infect Dis 47:1176-1184.

54. Maschmeyer G, Haas A, Cornely OA (2007) Invasive aspergillosis: epidemiology, diagnosis and management in immunocompromised patients. Drugs 67: 1567-1601.

55. Westney GE, Kesten S, De Hoyos A, Chapparro C, Winton T, et al. (1996) Aspergillus infection in single and double lung transplant recipients. Transplantation 61: 915- 919.

56. Singh N, Limaye AP, Forrest G (2006) Late onset invasive aspergillosis in organ transplant recipients in the current era. Med Mycol 44: 445-449.

57. Boch T, Reinwald M, Postina P (2015) Identification of invasive fungal diseases in immunocompromised patients by combining an Aspergillus specific PCR with a multifungal DNA-microarray from primary clinical samples. Mycoses 58: 735-745.

58. White PL, Barnes RA, Springer J (2015) EAPCRI. Clinical Performance of Aspergillus PCR for Testing Serum and Plasma: a Study by the European Aspergillus PCR Initiative. J Clin Microbiol 53: 2832-2837.

59. Imbert S, Gauthier L, Joly I (2016) PCR in serum for the diagnosis, follow-up and prognosis of invasive aspergillosis in neutropenic and nonneutropenic patients. Clin Microbiol Infect.

60. Arvanitis M, Anagnostou T, Mylonakis E (2015) Galactomannan and Polymerase Chain Reaction-Based Screening for Invasive Aspergillosis Among High-Risk Hematology Patients: A Diagnostic Meta-analysis. Clin Infect Dis 61:1263-1272.

61. Johnson GL, Sarker SJ, Nannini F (2015) Aspergillus-Specific Lateral-Flow Device and Real-Time PCR Testing of Bronchoalveolar Lavage Fluid: a Combination Biomarker Approach for Clinical Diagnosis of Invasive Pulmonary Aspergillosis. J Clin Microbiol 53: 2103-2108.

62. Herbrecht R, Denning DW, Patterson TF (2002) Invasive Fungal Infections Group of the European Organisation for Research and Treatment of Cancer and the Global Aspergillus Study Group. Voriconazole versus amphotericin B for primary therapy of invasive aspergillosis. N Engl J Med 347: 408-415. 
63. Lat A (2011) Thompson GR 3rd. Update on the optimal use of voriconazole for invasive fungal infections. Infect Drug Resist 4: 43-53.

64. Alexander BD, Perfect JR, Daly JS (2008) Posaconazole as salvage therapy in patients with invasive fungal infections after solid organ transplant. Transplantation 86: 791-796.

65. Hachem RY, Langston AA, Graybill JR (2008) Posaconazole as salvage treatment of invasive fungal infections in patients with underlying renal impairment. J Antimicrob Chemother 62: 1386-1391.

66. Pettit NN, Carver PL (2015) Isavuconazole: A New Option for the Management of Invasive Fungal Infections. Ann Pharmacother 49: 825-842.

67. Maertens JA, Raad II, Marr KA (2016) Isavuconazole versus voriconazole for primary treatment of invasive mould disease caused by Aspergillus and other filamentous fungi (SECURE): a phase 3 , randomised-controlled, non-inferiority trial. Lancet 387: 760-769.

68. Martín-Peña A, Aguilar-Guisado M, Espigado I, Cisneros JM (2014) Antifungal combination therapy for invasive aspergillosis. Clin Infect Dis 59: 1437-1445.

69. Marr KA, Schlamm HT, Herbrecht R (2015) Combination antifungal therapy for invasive aspergillosis: a randomized trial. Ann Intern Med 162: 81-89.

70. Rodriguez-Tudela JL, Berenguer J, Guarro J (2009) Epidemiology and outcome of Scedosporium prolificans infection, a review of 162 cases. Med Mycol 47: 359-370.

71. Idigoras P, Perez-Trallero E, Pineiro L (2001) Disseminated infection and colonization by Scedosporium prolificans: a review of 18 cases, 1990-1999. Clin Infect Dis 32: 158-165.

72. Wakabayashi Y, Okugawa S, Tatsuno K (2016) Scedosporium prolificansEndocarditis: Case Report and Literature Review. Intern Med 55: 79-82.

73. Johnson LS, Shields RK, Clancy CJ (2014) Epidemiology, clinical manifestations, and outcomes of Scedosporium infections among solid organ transplant recipients. Transpl Infect Dis 16: 578-587.

74. Cortez KJ, Roilides E, Quiroz-Telles F (2008) Infections caused by Scedosporium spp. Clin Microbiol Rev 21: 57-97.

75. Gilgado F, Cano J, Gene J, Serena C, Guarro J (2008) Different virulence of the species of the Pseudallescheria boydii complex. Med Mycol 24: 1-4.

76. Lamaris GA, Chamilos G, Lewis RE, Safdar A, Raad II, et al. (2006) Scedosporium infection in a tertiary care cancer center: a review of 25 cases from 1989-2006. Clin Infect Dis 43:1580-1584.

77. Caira M, Girmenia C, Valentini CG (2008) Scedosporiosis in patients with acute leukemia: a retrospective multicenter report. Haematologica 93: 104-110.

78. Husain S, Munoz P, Forrest G (2005) Infections due to Scedosporium apiospermum and Scedosporium prolificans in transplant recipients: clinical characteristics and impact of antifungal agent therapy on outcome. Clin Infect Dis 40: 89-99.

79. Cooley L, Spelman D, Thursky K, Slavin M (2007) Infection with Scedosporium apiospermum and S. prolificans, Australia. Emerg Infect Dis 13:1170-1177.

80. Castelli MV, Buitrago MJ, Bernal-Martinez L, Gomez-Lopez A, Rodriguez-Tudela JL, et al. (2008) Development and validation of a quantitative PCR assay for diagnosis of scedosporiosis. J Clin Microbiol 46: 3412-3416.

81. Sitterle E, Giraud S, Leto J (2014) Matrix-assisted laser desorption ionization-time of flight mass spectrometry for fast and accurate identification of Pseudallescheria/Scedosporium species. Clin Microbiol Infect 20: 929-935.

82. Gosbell IB, Toumasatos V, Yong J, Kuo RS, Ellis DH, et al. (2003) Cure of orthopaedic infection with Scedosporium prolificans, using voriconazole plus terbinafine, without the need for radical surgery. Mycoses 46: 233-236.

83. Mellinghoff IK, Winston DJ, Mukwaya G, Schiller GJ (2002) Treatment of Scedosporium apiospermum brain abscesses with posaconazole. Clin Infect Dis 34: 1648-1650.

84. Nesky MA, McDougal EC, Peacock Jr JE (2002) Pseudallescheria boydii brain abscess successfully treated with voriconazole and surgical drainage: case report and literature review of central nervous system pseudallescheriasis. Clin Infect Dis 31: 673-677.

85. Steinbach WJ, Perfect JR (2003) Scedosporium species infections and treatments. J Chemother 15: 16-27.

86. Troke P, Aguirrebengoa K, Arteaga C (2008) Global Scedosporium Study Group. Treatment of scedosporiosis with voriconazole: clinical experience with 107 patients. Antimicrob Agents Chemother 52: 1743-1750.

87. Trubiano JA, Paratz E, Wolf M, Teh BW, Todaro M, et al. (2014) Disseminated Scedosporium prolificans infection in an 'extensive metaboliser': navigating the minefield of drug interactions and pharmacogenomics. Mycoses 57: 572-576.

88. Hamaki T, Kami M, Kishi A (2004) Vesicles as initial skin manifestation of disseminated fusariosis after nonmyeloablative stem cell transplantation. Leuk Lymphoma 45: 631-633.

89. Cudillo L, Girmenia C, Santilli S (2005) Breakthrough fusariosis in a patient with acute lymphoblastic leukemia receiving voriconazole prophylaxis. Clin Infect Dis 40: 1212-1213.

90. Tezcan G, Ozhak-Baysan B, Alastruey-Izquierdo A (2009) Disseminated fusariosis caused by Fusarium verticillioides in an acute lymphoblastic leukemia patient after allogeneic hematopoietic stem cell transplantation. J Clin Microbiol 47: 278-281.

91. Dalyan Cilo B, Al-Hatmi AM, Seyedmousavi S (2015) Emergence of fusarioses in a university hospital in Turkey during a 20-year period. Eur J Clin Microbiol Infect Dis 34: 1683-1691.

92. Guarro J (2013) Fusariosis, a complex infection caused by a high diversity of fungal species refractory to treatment. Eur J Clin Microbiol Infect Dis 32: 1491-1500.

93. Nucci M, Anaissie E (2007) Fusarium infections in immunocompromised patients. Clin Microbiol Rev 20: 695-704.

94. Nucci M, Marr KA, Queiroz-Telles F (2004) Fusarium infection in hematopoietic stem cell transplant recipients. Clin Infect Dis 38: 1237-1242.

95. Garcia RR, Min Z, Narasimhan S, Bhanot N (2015) Fusarium brain abscess: case report and literature review. Mycoses 58: 22-26.

96. Stempel JM, Hammond SP, Sutton DA, Weiser LM, Marty FM (2015) Invasive Fusariosis in the Voriconazole Era: Single-Center 13-Year Experience. Open Forum Infect Dis 4: 2.

97. Nucci M, Anaissie EJ, Queiroz-Telles F (2003) Outcome predictors of 84 patients with hematologic malignancies and Fusarium infection. Cancer 98: 315-319. 
98. Van Diepeningen AD, Brankovics B, Iltes J, van der Lee TA (2015) Diagnosis of Fusarium Infections: Approaches to Identification by the Clinical Mycology Laboratory. Curr Fungal Infect Rep 9:135-143.

99. Triest D, Stubbe D, De Cremer K, Pierard D, Normand AC, et al. (2015) Use of matrix-assisted laser desorption ionization-time of flight mass spectrometry for identification of molds of the Fusarium genus. J Clin Microbiol 53: 465-476.

100. Gupta S, Almyroudis NG, Battiwalla M (2007) Successful treatment of disseminated fusariosis with posaconazole during neutropenia and subsequent allogeneic hematopoietic stem cell transplantation. Transpl Infect Dis 9:156-160.

101. Raad II, Hachem RY, Herbrecht R (2006) Posaconazole as salvage treatment for invasive fusariosis in patients with underlying hematologic malignancy and other conditions. Clin Infect Dis 2 : 1398-1403.

102. Ho DY, Lee JD, Rosso F, Montoya JG (2007) Treating disseminated fusariosis: amphotericin B, voriconazole or both? Mycoses 50: 227-231.

103. Neuburger S, Massenkeil G, Seibold M (2008) Successful salvage treatment of disseminated cutaneous fusariosis with liposomal amphotericin B and terbinafine after allogeneic stem cell transplantation. Transpl Infect Dis 10: 290-293.

104. Stelzmueller I, Lass-Floerl C, Geltner C (2008) Zygomycosis and other rare filamentous fungal infections in solid organ transplant recipients. Transpl Int 21: 534-546.

105. Spellberg B, Edwards J, Ibrahim A (2005) Novel perspectives on mucormycosis: pathophysiology, presentation, and management. Clinical microbiology reviews 18: 556-

106. Sun HY, Singh N (2009) Emerging importance of infections due to zygomycetes in organ transplant recipients. Int J Antimicrob Agents 32: 115-118.

107. Pang KA, Godet C, Fekkar A (2012) Breakthrough invasive mould infections in patients treated with caspofungin. J Infect 64: 424-429.

108. (2013) Diagnosis and treatment of mucormycosis in patients with hematological malignancies: guidelines from the $3 \mathrm{rd}$ European Conference on Infections in Leukemia (ECIL 3). Haematologica 98: 492-504.

109. Kasai M, Harrington SM, Francesconi A (2008) Detection of a molecular biomarker for zygomycetes by quantitative PCR assays of plasma, bronchoalveolar lavage, and lung tissue in a rabbit model of experimental pulmonary zygomycosis J Clin Microbiol 46: 3690-3702.

110. Vehreschild JJ, Birtel A, Vehreschild MJ, Liss B, Farowski F, et al. (2013) Mucormycosis treated with posaconazole: review of 96 case reports. Crit Rev Microbiol 39: 310-324.

111. Marty, Francisco M (2016) Isavuconazole treatment for mucormycosis: a single-arm open-label trial and case-control analysis. Lancet.

112. Spellberg B, Ibrahim A, Roilides E, Lewis RE, Lortholary O, et al. (2012) Combination therapy for mucormycosis: why, what, and how? Clin Infect Dis 1:73-78.

113. Spellberg B, Ibrahim AS, Chin-Hong PV, Kontoyiannis DP, Morris $\mathrm{MI}$, et al. (2012) The Deferasirox-AmBisome Therapy for Mucormycosis (DEFEAT Mucor) study: a randomized, doubleblinded, placebo-controlled trial. J Antimicrob Chemother 67: 715-722.
114. Adler A, Yaniv I, Samra Z, Yacobovich J, Fisher S, et al. (2006) Exserohilum: an emerging human pathogen. Eur J Clin Microbiol Infect Dis 25: 247-253.

115. Farina C, Gotti E, Parma A, Naldi L, Goglio A (2007) Pheohyphomycotic soft tissue disease caused by Alternaria alternata in a kidney transplant patient: a case report and literature review. Transplant Proc 39: 1655-1659.

116. Dubois D, Pihet M, Clech CL, Croue A, Beguin H, et al. (2005) Cutaneous phaeohyphomycosis due to Alternaria infectoria. Mycopathologia 160:117-123.

117. Osiyemi OO, Dowdy LM, Mallon SM, Cleary T (2001) Cerebral phaeohyphomycosis due to a novel species: report of a case and review of the literature. Transplantation 71: 1343-1346.

118. Revankar SG (2007) Dematiaceous fungi. Mycoses 50: 91-101.

119. Revankar SG, Jan E, Patterson, Deanna A, Sutton, et al. (2002) Disseminated Phaeohyphomycosis: review of an emerging mycosis. Clin Infect Dis 34: 467-476.

120. Purfield A, Cleveland AA, Benedict K, Harris JR, Brandt ME, et al. (2013) Multistate Fungal Infection Outbreak Response Team. Fungal infections associated with contaminated methylprednisolone injections. N Engl J Med 369: 1598-1609.

121. Brandt ME, Warnock DW (2003) Epidemiology, clinical manifestations, and therapy of infections caused by dematiaceous fungi. J Chemothe 2: 36-47.

122. Husain S, Wagener MM, Singh N (2001) Cryptococcus neoformans infection in organ transplant recipients: variables influencing clinical characteristics and outcome. Emerg Infect Dis 7: $375-381$

123. Vilchez RA, Fung J, Kusne S (2002) Cryptococcosis in organ transplant recipients: an overview. Am J Transplant 2: 575-580.

124. Singh N, Alexander BD, Lortholary O (2007) Cryptococcus neoformans in organ transplant recipients: impact of calcineurin-inhibitor agents on mortality. J Infect Dis 195:756-764.

125. Shaariah W, Morad Z, Suleiman AB (1992) Cryptococcosis in renal transplant recipients. Transplant Proc 24: 1898-1899.

126. Singh N, Dromer F, Perfect JR (2008) Cryptococcosis in solid organ transplant recipients: current state of the science. Clin Infect Dis 47: 1321-1327.

127. Aberg JA, Mundy LM, Powderly WG (1999) Pulmonary cryptococcosis in patients without HIV infection. Chest 115: 734-740.

128. Singh N, Alexander BD, Lortholary O (2008) Pulmonary cryptococcosis in solid organ transplant recipients: clinical relevance of serum cryptococcal antigen. Clin Infect Dis 46: 12-18.

129. Singh N, Dromer F, Perfect JR (2008) Cryptococcosis in solid organ transplant recipients: current state of the science. Clin Infect Dis 47: 1321-1327.

130. Singh N, Gayowski T, Marino IR (1998) Successful treatment of disseminated cryptococcosis in a liver transplant recipient with fluconazole and flucytosine, an all oral regimen. Transpl Int 11: 63-65.

131. Singh N, Lortholary O, Alexander BD (2005) Antifungal management practices and evolution of infection in organ transplant recipients with Cryptococcus neoformans infection. Transplantation 80: 1033-1039. 
132. Macsween KF, Bicanic T, Brouwer AE (2005) Lumbar drainage for control of raised cerebrospinal fluid pressure in cryptococcal meningitis: case report and review. J Infect 51: e221-e224.

133. Speed B, Dunt D (1995) Clinical and host differences between infections with the two varieties of Cryptococcus neoformans. Clin Infect Dis 21:28-34; discussion 35-36.

134. Sorrell TC, Allworth AM (1995) Cryptococcal disease of the CNS in immunocompetent hosts: influence of cryptococcal variety on clinical manifestations and outcome. Clin Infect Dis 20: 611-616.
135. Thompson GR 3rd, Wiederhold NP, Fothergill AW (2009) Antifungal susceptibilities among different serotypes of Cryptococcus gattii and Cryptococcus neoformans. Antimicrob Agents Chemother 53: 309-311. 